\title{
Complexity in simple tasks: a qualitative analysis of GPs' completion of long-term incapacity forms
}

\author{
Julia Hiscock ${ }^{1}$, Paula Byrne ${ }^{2}$, Sarah Peters ${ }^{3}$, Debra Westlake ${ }^{4}$ and Mark Gabbay ${ }^{2}$ \\ ${ }^{1}$ NPCRDC, University of Manchester, Manchester, UK \\ ${ }^{2}$ School of Population, Community and Behavioural sciences, University of Liverpool, Liverpool, UK \\ ${ }^{3}$ School of Psychological Sciences, University of Manchester, Manchester, UK \\ ${ }^{4}$ Mersey Primary Care R\&D Consortium, Division of Primary Care, University of Liverpool, Liverpool, UK
}

\begin{abstract}
Aim: To explore the factors influencing the completion of the IB113 form for the Department for Work and Pensions (DWP), as an exemplar of how general practitioners (GPs) manage and report patient information to external bodies. Background: In UK, GPs complete IB113 forms for their patients approaching longer-term sickness absence, who may be exempt from the incapacity benefit linked medical examination. The DWP has expressed concerns about the quality of such reports, and GP organizations have raised objections to completing such forms. The content of returned forms is variable, and may be subject to a number of influences. Design: Qualitative interviews with purposive sampling of GPs and practice managers (PMs). Setting: Primary Care practices in the North East of England. Method: GPs and PMs were interviewed using a semi-structured topic guide about completing IB113 forms for the DWP about their patients entering long-term incapacity. The transcribed data were analysed thematically using the framework analysis method. Results: Whilst the IB113 appears superficially straightforward to complete, our results demonstrate levels of overlapping complexity that add ranges of subjectivity and selectivity onto factual reporting, including practice protocols, the gathering and managing of information, the doctor-patient relationship, and doctor's personal views on systems. Conclusions: The recording and reporting of patient related data by GPs is subject to complex influences, which need to be understood and managed to improve the relevance and quality of reports to third parties.
\end{abstract}

Key words: completion of long-term incapacity forms; general practitioners; qualitative

Received 22 September 2008; accepted 1 May 2009

\section{Introduction}

General Practitioners (GPs) have been providing evidence to employers about their workers' fitness since the mid-nineteenth century. Before the

Correspondence to: Paula Byrne, School of Population, Community and Behavioural sciences, University of Liverpool, Brownlow Hill, Liverpool L69 3G, UK. Email: paula. byrne@liv.ac.uk

(C) 2009 Cambridge University Press
NHS was established, caring for, and maintaining a fit workforce, was often a major source of income for GPs; for example, the colliery doctors during the nineteenth and early twentieth century (Digby, 1999). However, with the introduction of the National Health Insurance scheme with patient panels in 1911, and the creation of the NHS in 1948 with its government sick-note system for GPs, the role of the state was strengthened. GPs, rather than local employers, became 
the focus of sickness certification decisions, leading to split loyalty for GPs (Digby, 1999). The quality of the relationship between the GP and patients, linked to an advocacy role on their behalf, lies at the heart of General Practice philosophy and training (Pendleton et al., 1984; Morrell, 1991; Purves, 2002; Hull, 2003; Neighbour, 2005). Thus, when completing forms for third parties about their patients, GPs are often the servants of two masters.

General practitioners in the UK are required, as part of their contract, to provide a range of medical evidence for the Department for Work and Pensions (DWP) (Department for Work and Pensions, 2004). For short-term sickness absence (over seven days but less than six months), GPs complete medical certificates certifying whether their patient is fit for work. As the potential switch to incapacity benefit after 26 weeks of sickness approaches, GPs complete a more detailed certificate - MED 4. On reviewing this, Benefits Officers (at the DWP) may consider that the claimant meets the qualification for incapacity without the requirement for the personal capability assessment (an independent medical examination by a DWP services doctor). The IB113 is then sent to GPs by the DWP, requesting medical and functionality information about patients being assessed for incapacity benefit, to provide evidence in regard to the exemption decision. GPs are required to complete this form within their NHS contract.

Patients are seeking a transfer onto incapacity benefit, which obviates the need for regular sickness certification, gives access to longer-term benefits or insurance, and if unemployed, the requirement to regularly attend the benefits office and apply for work. A recent DWP strategy document however, recommends a substantial rethinking of welfare versus employment for those with sickness and disability, suggesting that one million claimants currently on incapacity and sickness benefits could return to paid employment (DWP, 2005).

The DWP seeks what they believe to be the GP's medical and personal knowledge of their patient through the IB113, focusing on diagnosis, management and the impact of the illness on function and capacity. If the claim is successful, GPs are only required to provide further evidence when requested by the DWP when incapacity is being reviewed. The 1B204 guide for GPs completing IB113 states 'a fully completed report may avoid the need for the patient to undergo a benefit related examination, helps Benefits Agency give a prompt decision on their entitlement and reduces the number of requests for reports in the event of an appeal...' (DWP, 2004).

General Practice prides itself on increasingly computerized, sophisticated and comprehensive patient records systems (Neary, 2003), which, alongside the breadth and depth of information practice staff have about their patients, are considered a rich source of expert data (Hull, 2003). One might anticipate that form completion should be a relatively straightforward task in such circumstances, as it is for forms for insurance companies much of which is now computerized. However, a substantial proportion of IB113 forms are returned late or not at all. Furthermore, the information provided may be scanty or considered to be of poor quality (Sainsbury et al., 2003). One possible interpretation may suggest that completing apparently simple forms may involve unanticipated complexities.

Previous research suggests that there have been some tensions surrounding the GP's role in medical certification, including difficulties in deciding the length of sick leave, problems interpreting the benefit rules, dilemmas in making decisions about the functionality of a patient in relation to their ability to work and everyday activities and the lack of evidence or appropriate knowledge base by which GPs can make such decisions (Hussey et al., 2003; Soderberg et al., 2003; Wahlstrom and Alexanderson, 2004). Other research suggests that GPs are influenced in their provision of medical evidence by a wide range of factors (Hiscock and Ritchie, 2001; Hussey et al., 2003), which go beyond diagnosis and co-morbidity, often including the family and professional environment (Elston et al., 2002), the GP-patient relationship (Wahlstrom and Alexanderson, 2004), concerns about the potential for conflict (Elston et al., 2002) and feeling coerced (Mayhew and Nordlund, 1988; Elston et al., 2002). A recent study of IB113 completion suggests that GPs provide more useful evidence than information found from patients' medical records or third parties (Sainsbury et al., 2003). Such findings suggest that some GPs negotiate with the 'facts' in patient's medical records along with their own situated knowledge about patients, which they 


\section{IB113 questions}

1. Date patient was last seen or examined for the condition(s) causing incapacity

2. Diagnosis of all relevant conditions and date(s) of onset

3. Factual details of patient's condition, where possible, please include brief factual details of: present medical condition, medication and other treatments (eg attendance at daycare centre, hospital outpatient), outlook for your patient and any proposals for future management.

4. Where available to you, please give brief details of what the patient has been told about the likely clinical course of their condition(s), and any future treatment.

5. Any other information- if you have evidence which indicates that, as a result of their medical condition, your patient would not be able to attend an examination by using public transport or by taxi please include this here. Any additional information about the effects of the medical conditions on functionality (self care, indoor mobility, judgement and compliance with medication) would be very helpful.

6. If you have diagnosed a psychiatric condition- where available to you, please give brief details of any history of recent or serious attempts at suicide or other self injury, or any history of threatening or violent behaviour towards others.

There is an option to withhold evidence that would be harmful to the patient's health, which should then be provided separately.

Figure 1 IB113 questions

attempt to communicate in the IB113, rather than simply presenting diagnostic categories.

This paper presents some of our findings from a DWP commissioned project (Hiscock et al., 2005). Our aim was to contribute to the knowledge base about the work undertaken by GPs and practice managers (PMs) in the provision of medical evidence, specifically, to explore the factors influencing the completion of the IB113 form for the DWP. Such work can be viewed as relevant and applicable to the role of GPs and primary care practices in the provision of medical evidence in general, including consideration of broader aspects of culture, attitudes and relationships. We focused on the IB113 in particular (as part of an evaluation of a revised IB113 form), as an illustration of how information about patients is represented to external agencies such as the DWP (see Figure 1). In this paper, we explore the complexity of the GPs task to provide 'medical evidence' for incapacity benefit, including the tactics and strategies they use to manage and respond to these tensions. Thus, we hope to suggest how these factors may be ameliorated to manage such tasks, improving both completion rates and quality of information.

\section{Methods}

We used a qualitative approach to obtain the depth of data required to explore knowledge, attitudes and behaviour of the GPs and PMs. Our team included one GP, one psychologist and three social scientists from differing backgrounds (Hiscock et al., 2005). All members of the research team are experienced qualitative researchers in health research and carried out the 43 interviews between them. The team members were chosen for their relatively equivalent experience of qualitative methods, and their understanding of primary care. Our study had local ethical and research governance approval and was carried out in the autumn/winter of 2004.

Our topic guide (Appendix 1) was based on a literature review, and a group discussion between team members, GPs, nursing and practice administrative staff known to the research team. This was refined following further discussions about the policy contexts with the DWP research team.

In all, 25 semi-structured interviews were conducted with GPs and 18 with PMs from practices in four geographically distinct areas in North East England where the DWP's modified IB113 form was being piloted. Purposive sampling was used to identify a range of GP and PM respondents, providing a spread of practice, practitioner and catchment area characteristics (Hiscock et al., 2005). The majority of practices involved had four to six GPs, with one single-handed GP, and three practices with over 10 GPs working within the practices. Twenty-two of the GPs had been qualified for more than six years, with eight practising for more than 21 years. The practice list sizes were varied, with eight serving 10000-15000, and two of the practices with a list size over 20000 (see Table 1). One GP was currently working in Medical Services, and two others had previously been involved.

As all of the interviews were semi-structured, respondents were interviewed once; interviews typically lasted 30-40 min. A total of 108 primary care practices were approached, and the sample was drawn from those volunteering to be interviewed (see Table 2). Interviews were arranged by an administrative member of the team. All interviews were carried out in the primary care practices where the GPs and PMs worked. 
Table 1 Practice characteristics

\begin{tabular}{lr}
\hline List size of practices & \\
4999 and under & 4 \\
$5000-9999$ & 8 \\
$10000-14999$ & 8 \\
$15000-19999$ & $?$ \\
20000 and over & 24 \\
Total & \\
Number of GPs in practices & 1 \\
Single-handed & 3 \\
$2-3$ & 10 \\
$4-6$ & 7 \\
$7-9$ & 2 \\
$10-12$ & 1 \\
13 and over & $\mathbf{2 4}$ \\
Total & \\
\hline
\end{tabular}

$\mathrm{GPs}=$ general practitioners.

Table 2 Respondent characteristics

\begin{tabular}{lcc}
\hline & GPs & PMs \\
\hline 0-5 years in practice & 3 & 2 \\
6-10 years in practice & 6 & 4 \\
11-20 years in practice & 8 & 8 \\
21-30 years in practice & 6 & 4 \\
Over 30 years in practice & 2 & 0 \\
Total & $\mathbf{2 5}$ & $\mathbf{1 8}$ \\
Number of males & 21 & 3 \\
\hline
\end{tabular}

$\mathrm{GPs}=$ general practitioners; $\mathrm{PMs}=$ practice managers.

We used the manual matrix method: 'Framework' to organize, summarize and analyse the transcribed interviews. The Framework approach is particularly suited to policy related qualitative research, as this frequently involves a short research period and a focused approach. Thus, whilst it is grounded within an inductive philosophy, it relates the material to pre-determined ideas. The data analysis has five phases: familiarization, thematic framework development, indexing, charting, mapping and interpreting. Members of the research team were involved at different stages of the analysis. An Excel database was set up with each column representing a question from the Topic Guide; data from each interview was then imported into the relevant column following discussion amongst the researchers.

\section{Results}

We traced in detail the pathway in primary care practices of a 'simple form', the IB113, to uncover the systems and factors that influence its completion. The main themes which emerged and had a significant effect upon it were: the practice protocols within each practice; the information gathering by GPs to fill in the form; completing the form/managing information for the purposes of the IB113; the impact of filling in the form on the doctor-patient relationship and; the influence of the world view/personal views of GPs on the benefit systems.

\section{Practice protocols}

Complexity begins when the IB113 arrives at a practice. PMs and GPs described elaborate administrative systems for logging in and tracking the path of the IB113 from arrival to return (or not) to the DWP. In practices with established audit trails of incoming correspondence, form IB113 is smoothly incorporated into the system.

We've got procedures for most things, including what we do when this form arrives here... . On the day form arrives it's logged on the record, we scan all incoming post and attach it to the patient records, we've set up a template in patient records, within the template there's a procedure to follow, not just for this form, but for all forms, fee generating and not.

(078c PM)

Form comes in, we put it straight on the computer, so we bring the patient's name up and then code it on the patient's record with the code which means IB113, when we put that code on the computer it will come up as incapacity for work... We don't like things like this hanging around for long that's why we like things to be done on a daily basis, we like to do today's work today, that's our little code.

(055b PM)

Such protocols were linked to the role of the PM rather than whether paperless or paper-light. In many cases, administrative staff and GPs also engaged in a process of 'redirecting' the IB113 forms to the most appropriate GP since IB113s were often not addressed to the GP most familiar with the patient.

Our rule is that it goes to the doctor who sees that patient. The other rule that's applied 
quite strictly is that if it's a patient lots of doctors have seen, the last doctor [to have seen] the patient will do the report.

(019a PM)

Other practices did not track the form or monitor its progress in the practice.

No, doesn't pass in front of me, comes in the post, opened by one of the girls in reception, who'd see who sees the patient most by going into the clinical system and just popped in the doctors docket on the wall to fill in... . Don't see the forms after completion as doctors normally put them in the pre-paid envelope...completed forms are not logged in in any way...it's not something we're concerned with, what sorts of benefits they're receiving.

(036b PM)

Thus PMs oversee administrative procedures that vary in their sophistication, and represent the heterogeneity of practice information management systems.

\section{Information gathering}

The IB113 (unlike sickness certificates) is completed outside of the consultation framework. GPs rely on records (paper, electronic or both) and/or memory as their sources of information.

I'll run a computerised printout for medication...you're going between the computer, your computerised notes and your knowledge of the patient.

(005a GP)

Some of it is flicking through the notes, it asks for all relevant conditions and some of them are things the patient has indicated themselves and occasionally you have to go back, 'cos sometimes people have chronic conditions that go on for a long time and are so chronic that they're not always indicated in the notes, but sometimes you have a references point which jogs your memory.

(003a GP)

If it's a patient I know well it's easy and I almost do them from memory, but if it's a patient I don't know well, then I have to look at the notes...extracting from notes, especially computerised notes is not always easy.

(078 GP)

Occasionally, GPs called in a patient to ensure that information recorded on to the IB113 was correct.

If I'm not sure from records I'll call the patient in, regardless of the fact that it says on them you do not need to see or examine this patient. I'm not going to sign the form on their behalf that's going to either give them benefit where appropriate...I should be doing the job properly and if that means seeing my patient, that's what I'll do.

(066bz GP)

General practitioners complained that record trawling for the required information could be time-consuming and potentially frustrating, as the relevant information was often not recorded.

The dreaded IB113! Can sit in tray for a week or so and then have to be done, difficult to fill in as GP has to refer to notes and they take a long time to fill in, can't do the form from memory.

(011a GP)

Whilst others were perhaps more relaxed about it:

Fill in from the computer what is there...sometimes when you're busy and haven't got much time, it's difficult to put all the detail. Sometimes you just put the main points as it's required to do so.

(029a GP)

Clinicians rarely recorded information about patients 'functionality'.

Even if you know the patient and maybe tracked their illness, the most difficult ones are multiple illnesses and has been seen by multiple doctors with psychiatric overtones or personality problems, grey type conditions...Sometimes may not have the information about functional daily living.

(033b GP)

The competing demands between factual information and functionality, which the IB113 asks for, are particularly difficult for GPs to manage. 


\section{Completing the form/managing information}

Further complexity is introduced when GPs begin to consider what to write on the form, which is influenced by a number of factors such as, prioritization of work, time, and competing demands:

Time pressures, simple as that...'cos we get a batch of paperwork you know each week... and my enjoyment of the job is seeing patients and talking to them and managing their problems and not the paperwork thing, is kind of very low on my priorities...the things that I have to do, like insurance reports and Disability Living Allowance forms, in the scale of things seem much more important, and so they take priority.

(024a GP)

I fill these forms in a crap way...it's just a chore... I get away with the bare minimum.

(036b GP)

Dilemmas caused by trying to match available information to the form's requirements:

Myself, and I suspect most people, answer question 3 as briefly as possible even though it's a huge box. It's usually brief because we don't really know the answers to the questions... The information required by the form is very difficult to fish out, diagnosis and date of onset, for some people that's a long list and dates of onset are very difficult to find accurately.

(019a GP)

General practitioners also described the form's expectations as ambiguous. The IB113 form asks for patient details, a list of diagnoses and 'factual details of patient's condition', supplemented by what the patient's been told about their prognosis and treatment, and, if relevant, a brief mental health risk assessment.

Number 3 [Question 3 on the IB113, see Figure 1] is quite difficult as well. If you haven't seen them for a while you can't actually comment on the person's medical condition, you don't know what to write there because you don't know. If I have seen the patient the last time in 2002 you can't say what their present medication condition is...still number 3, you are supposed to comment on the outlook for the patient and again it's difficult to write if you are no expert.

(097b GP)

It's factual information that they need and it's factual information that you put on the form. You don't consider the emotions of the patient, I don't consider the emotions of the patient when I'm completing the form, if I do that my judgement would be wrong in that I would just start filling in things that are not there.

(084c GP)

Despite this, some GPs felt strongly that completing the form required opinions:

Well, I mean asking about a prognosis for example, I mean that's not a fact, that's a prediction isn't it? So there it's not just pure facts they're after, I guess they're looking for something else, opinion as well, an outlook for the patient. 'Present medical condition', that's a kind of opinion isn't it, how they are at the moment? Two doctors could disagree about that I'm sure. So, it's not a fact, it's an opinion, but I'm sure they want us to keep it as factual as possible.

(019a GP)

Again, GPs struggled with the tension between the 'facts' as they saw them and the demands of the DWP for information, which GPs saw as outside of their remit.

\section{The doctor-patient relationship}

This was a significant factor in the type of information recorded on the IB113, and at times was dependent upon GPs' attitude towards a patient's psychological status and his or her ability to work.

Subconsciously [it] may be patient factors that influence how to fill forms, depending on relationship with patient, if it's a patient you can't stand and you're seeing them every two weeks, I'd may be adversely influence and even say 'I think this patient should be back at work'... It would be my honest opinion... I think I'm more sympathetic to patients with serious physical diseases rather than those with psychological problems, my empathy is 
less with them than with those with a definite pathology.

(078 GP)

I do think that there are patients where its very obviously that they're not fit for work... and really we should be able to, pardon my French, but cut the crap, and really not hassle these patients.

(075bz GP)

General practitioners were concerned that providing medical evidence to the DWP had the potential to cause conflict, mistrust or unpleasant exchanges between themselves and their patients. GPs wanted to avoid all of this. They developed three main coping strategies. First, they tended to avoid challenging the patient:

It bothers me that it disrupts my relationship with my patients to have that adjudicative role as well as the advocative role that I'm more comfortable with.

(076a GP)

Secondly, they filled in the IB113 in such a way that passed responsibility back to the DWP for assessment of fitness to work:

If the patient is trying to fiddle the system, right, you give factual information. The doctor at the Jobcentre Plus will also read what's in print and from there they will judge.

(048c GP)

Thirdly, where GPs did wish to convey a message to the DWP about a patient's fitness for work, they used 'codes':

I'd use words like 'genuine' and things along those lines to stress that, you know it's a genuine problem... and then for others I imagine what we write isn't very helpful and probably doesn't contribute to it a great deal, they're the ones we don't really know. And then there are some I suppose where it's very difficult to suggest that they're not incapacitated, but rarely, there might have been times where I've done that as well, you know, maybe pointing out that, in section 3, saying how well they've improved and things like that.

(019aGP)

General practitioners used 'codes' in cases where their view differed from the patient's view, in order to avoid directly challenging the patient and to protect the doctor-patient relationship. They used subtle mechanisms to communicate with the DWP via the IB113 form, namely using pointed phrases such as 'improved'; they made it clear that evidence was based on patient's narratives, for example:

...they come to us, 'Can you give a note that I can't travel?' I say 'listen, I only fill this form, you write to the department' And if they insist I write here... 'Patient has asked me to write that he can't travel' 'he asked me to write'.

(026aGP)

This signifies to the reader that they may not agree with such a statement; using omission as a strategy by 'skimping' on some sections or leaving sections blank, to indicate that the patient needed to be assessed by the Medical Officer employed by the Benefits Agency. One GP reflected: 'whether people at the other end pick up on the subtlety of that I do not know'.

Furthermore, alongside the use of codes to their unknown colleague, GPs were aware that patients might read what they wrote and this might impact on the relationship.

It's actually quite complex what you're doing then as well, coz in your head while you're filling in, you're thinking about the patient, if they read this, and you've got this other kind of more subtle role sometimes of thinking well, is that doctor going to understand what I'm trying to say as well.

(075bz GP)

In addition to guarding the doctor-patient relationship, GPs were aware of their concurrent obligations to the DWP, and the wider implications towards society; being the 'servant of two masters' often caused ambivalence and frustration.

I struggle with this because you know when I was new into the system as a GP I think I had a...I mean you know, I guess my basic slant on the world is a lot of extremely distressed and unhappy people who don't get the support and help that they need, and that is so much bigger a problem than people who get more support and help than they need, you know our attention ought to be focused on the former.

(102c GP) 
In the example above, the GP is articulating a more political slant, whereby the focus of the State is diverted away from what $\mathrm{s} / \mathrm{he}$ considers to be perceived real need, and individuals who already have in place extra support, such as extra benefits.

Indeed, GPs were at pains to present a representation of their patients that could be judged to be fair and objective.

Right, so the way I'm thinking or the way I see it is you're really trying to tell a story about your patient aren't you, relevant information is included in the story to support your responsibility towards that patient...it has to be something that if the patient read, that they could accept it as a fair and accurate reflection...it has to be independent to an extent in a sense that you know that I won't miss out relevant positives.

(075bz GP)

\section{World view/personal views on systems}

General practitioners personal beliefs and attitudes towards work and illness were contributory factors in completing the IB113. Despite some GPs' assurance that the completion of the IB113 was guided by other factors, the strength of an individual's own experiences and socialization as a medical doctor and member of a family, had a direct impact upon GPs' perceptions and treatment of individuals.

Most doctors have a very strong work ethic...most don't take time off ourselves, so people shirking are not something that is familiar to us or part of our direct experience...some people have a large number of days off. That is not how I was brought up. It is not how my family operates.

(058c GP)

It probably is something that...I was thinking when the registrars come, I mean I just show the registrars how to fill the forms in...as I say the difficulty is how much personal feeling you have about advocacy... is probably the biggest factor in that effects what is actually written down.

(003a GP)
The limitations of GPs in relation to structural inequalities, such as poverty, are illustrated in the quote above. Some GPs recognize that the ability of financially deprived individuals to manage their lives can be severely curtailed, against a backdrop of the psychological benefits from being in work.

We do our best to try and sort out what, there are a lot of unhappy people around, there are a lot of social circumstances which are, there is poverty, how do you sort that out? I think as a practice we avoid medicalising and encourage people to be in work if possible because I think that's beneficial on the whole...it can work two ways, maybe you can't face work, but on the other hand some people sitting at home all day isn't good for them either.

(110c GP)

I mean, I think I'm probably at that end of the spectrum of GPs where I'm more concerned about people who are being inadequately supported by the Welfare State than I am personally about people who are being over supported by the Welfare State, just because I encounter a lot more of the former, and the consequences are a lot more serious...I try and fill in the form in a way that does justice to their needs and to their disabilities.

(102c GP)

The moral and ethical issues of GPs being placed as gatekeepers to the benefits' system and their obligations, or advocacy role for their patients was a feature which was often raised in our interviews.

\section{Discussion}

\section{Summary of main findings}

As we have shown, the interplay between GPs, patient's requests and state benefits are challenging and highlight the crucial role that primary care provides within the wider society. Most practices describe effective protocols and systems for managing and tracking forms and information management linked to robust information technology systems. However, GPs' manual completion

Primary Health Care Research \& Development 2009; 10: 254-269 
of the IB113 is subject to a variety of factors that illuminate this apparently simple task as complex. At the heart of this complexity are several issues. First, the different types of information required by the DWP, both 'factual' about illnesses and diseases, and more problematically, 'functionality' where GPs are placed in hypothetical situations. These two types of information call upon GPs' own objective and subjective knowledge about a patient. Secondly, and related to these types of knowledge, is the changing relationship between GPs and their patients. Whereas previously, the intimate knowledge about a patient, their family and circumstances was routinely known by GPs, continuity of care - a traditional cornerstone of practice - is less of a priority within a target-led and externally managed approach. Thirdly, the IB113 represents a GP's gate-keeping role, allowing a patient into long-term sickness and all the concomitant access into benefits, and withdrawal from work.

\section{Objective/subjective}

Our evidence suggests, however, that the key to complexity is a mismatch between the objective information the DWP requests, and the frequently more subjective approach taken when completing the form, not merely simplifying the means to provide extracts from records. This resonates with the findings from a previous pilot where records were directly provided, but problems with form completion persisted (Sainsbury et al., 2003). GPs describe a variety of personal views and opinions that influence how they approach the form in general, and the information they provide on individuals. This may be partly due to aspects of the information requested that are not routinely recorded, such as patient functionality, and soft information such as prognosis. Indeed, within Primary Care such information is likely to be relatively subjective, as there is no widespread commonly practiced functionality assessment used in General Practice, and GPs are much less likely than in the past to see patients in their own homes unless they are bedbound. Only a proportion of claimants will have been seen by a specialist, and few provide specific opinions on prognosis. These issues need to be considered, as the emphasis for UK sick note certification follows the UK five-year strategy-driven policy, of a shift from incapacity assessment to capacity for work. It may well be that other health professionals such as medical specialists, district nurses, occupational therapists and physiotherapists may be better placed to offer opinions on functionality, rather than GPs. The expertise of GPs may be more usefully employed to offer diagnostic and medical therapy information.

\section{Changing relationships}

Additionally, the results from this study also reveal the ambivalence of working for the benefit of their patients, their state employer or contractor and wider society. The DWP considers GPs the best source of such background information to inform their decision-making. However, GP's recollections of the patient were frequently the key source of information, which may be faster, but may be subject to recall bias and inaccuracies. This approach presupposes that GPs have an in-depth and personal knowledge of each patient. This may or may not have been the case in the past, but it is increasingly less comprehensive in contemporary primary care where GPs no longer carry personal lists and patients often see a variety of clinicians in larger teams, to meet appointment access targets. GPs thus have to increasingly supplement such knowledge with record reviews and conversations with colleagues.

\section{Gate-keeping role}

We also provide evidence of different responses between practitioners and by some practitioners under different circumstances. While some GPs take the forms very seriously and complete them with care, others place a low priority on their completion seeing them as a 'chore', with one GP describing the form as 'an evil'. Part of a GP's job is to control and manage information, but the IB113 forces GPs to make judgements about patients, whether these are conscious or unconscious, on their appropriateness to be 'long-term sick' and also allowing them access into a specific part of the benefits system. Such judgements are in contrast to the bulk of GPs' work which involves the management of patients' symptoms, dealing with clinical uncertainty, negotiating management plans and gate-keeping access to additional services. It possibly also reflects GPs perceived role as coordinators and gatekeepers of 
care for their patients, which includes considerations of costs to the NHS. They are increasingly being invited to influence NHS spending decisions more widely, as exampled by practice-based commissioning, and in recent history GP fund-holding. It is perhaps unsurprising that such thinking may influence their completion of forms about incapacity benefit. The bottom line for patients is that the GP can influence their access to welfare benefit and GPs are aware of their role, the consequences to the individual and their relationship with them, as well as the socio-economic implications.

\section{Implications for future research and clinical practice}

Most contemporary and recent patient information is now held electronically, making the completion of a paper form more complex and convoluted. In contrast, many forms or letters are completed semi-automatically within practices, and information is distributed via e-mail or internal websites. A 2007 DWP IB113 form pilot in the North West of England allowed GPs to print out relevant sections of the electronic patient record and attach this to the form. Thus, GPs only have to add any specific items missing from this printout. It also asks about any planned or existing workplace adjustments affecting the condition. A detailed section on patient function remains. Our study opens up some interesting areas for exploring how practitioners make decisions and undertake complex tasks, even if, on the face of it, they appear simple. Learning how to make a decision as a GP is influenced by a multitude of factors: personal attitudes, the type and quality of information held in a medical record, perceptions about a patient, negotiating a relationship with a patient, and tacit and implicit knowledge within communities (Gabbay and le May, 2004). Further research would help understand how GPs manage the complexity of information within their professional influences and personal attitudes to improve the way that such information is gathered, and to improve the consistency of its reporting to third parties. When designing forms for collecting data from GPs that extend beyond direct data extraction, those requesting the information need to understand that where information is not directly available from the record, a complex variety of factors may influence the content of responses. GPs completing reports need to be alert to their own tendency to colour their responses with subjective statements influenced by their personal attitudes and opinions.

\section{Strengths and limitations of the study}

We used qualitative methods to gain in-depth information on the processes and behaviour required for our objectives. We had a wellbalanced, relatively large purposive sample for a study of this type. The analysis was undertaken by a range of researchers from different disciplines, which gave breadth and robustness to our results. However, we cannot verify the scale and extent of the behaviours described or their influence in 'real-life' form completion. We focused on one region of the UK (though on four differing areas within it), covering a variety of demographic and practice features.

This study was commissioned by the DWP to evaluate their IB113 pilot. The findings presented in this paper, however, largely relate to data gathered from interviews that were more global in their focus than the pilot itself. We were in regular contact with DWP R\&D staff during the project, who made observations and suggestions. However, we maintained academic and intellectual independence throughout.

\section{Comparison with existing literature}

Other studies (Hussey et al., 2003; Shiels and Gabbay, 2007) have examined GPs' role in provision of evidence for short-term sickness absence certification. There are similarities in the attitudes and behaviour of GPs in provision of medical evidence for short- and long-term incapacity benefit. The only other study to examine the IB113 form concluded that in comparison to other options, a well completed IB113 by a GP is the best way to obtain medical information for incapacity benefit, but often fell short of this standard (Sainsbury et al., 2003). Our study adds to this; it illustrates the complexity of factors that may prevent IB113s from meeting the needs of the DWP officers for specific information to inform their decision-making. 


\section{Acknowledgements}

This study was funded through the DWP social and economic research framework. Ethical approval for this study was granted by the West Hertfordshire MREC (04/Q0203/35). There are no competing interests to declare for any authors. The authors acknowledge the help of DWP research staff in developing this study, the practitioners and managers who agreed to take part, and administrative support of colleagues within the research consortium.

\section{References}

Department for Work and Pensions. 2004: Section 5: Medical reports for incapacity benefits. In Medical evidence for statutory sick pay, statutory maternity pay and social security incapacity benefit purposes: a guide for registered medical practitioners. Norwich: HMSO.

Department for Work and Pensions. 2005: Supporting people with health conditions and disabilities. Ch. 4. In Five year strategy. Opportunity and security throughout life, Cm 6447. Norwich: HMSO, 40-51.

Digby, A. 1999: The evolution of British General Practice 1850-1948. Oxford: Oxford University Press.

Elston, M.A., Gabe, J., Denney, D., Lee, R. and O'Beirne, M. 2002: Violence against doctors: a medical(ised) problem? The case of national health service general practitioners. Sociology of Health and Illness 24, 275-98.

Gabbay, J. and le May, A. 2004: Evidence based guidelines or collectively constructed 'mindlines?' Ethnographic study of knowledge management in primary care. British Medical Journal 329, 1013-14.

Hiscock, J., Hodgson, P., Peters, S., Westlake, D. and Gabbay, M. 2005: Engaging physicians benefiting patients: a qualitative study. DWP research report no. 256. London: DWP.
Hiscock, J. and Ritchie, J. 2001: The role of GPs in sickness certification. DWP research report no. 148. Leeds: Corporate Document Services.

Hull, S. 2003: Celebrating the study of the doctor-patient relationship. In Lakhani, M., editor, A celebration of general practice. Oxford: Radcliffe Medical Press.

Hussey, S., Hoddinott, P., Wilson, P., Dowell, J. and Barbour, R. 2003: Sickness certification system in the United Kingdom: qualitative study of views of general practitioners in Scotland. British Medical Journal 328, 88-93.

Mayhew, H.E. and Nordlund, D.J. 1988: Absenteeism certification: the physician's role. Journal of Family Practice 26, 651-55.

Morrell, D. 1991: The art of General Practice. Oxford: Oxford University Press.

Neary, J. 2003: Achievements in record keeping: the use of information management and technology. In Lakhani, M., editor, A celebration of General Practice. Oxford: Radcliffe Medical Press.

Neighbour, R. 2005: The inner consultation, second edition. Oxford: Radcliffe Medical Press.

Pendleton, D., Schofield, T., Tate, P. and Havelock, P. 1984: The consultation: an approach to learning and teaching. Oxford: Radcliffe Medical Press.

Purves, I. 2002: The changing consultation. In Harrison, J. and van Zwannenberg, T., editors, GP Tomorrow, second Edition. Oxford: Radcliffe Medical Press.

Sainsbury, R., Corden, A. and Finch, N. 2003: Medical evidence and incapacity benefit: evaluation of a pilot study. Research report no. 189. London: DWP.

Shiels, C. and Gabbay, M.B. 2007: Patient, clinician and general practice factors in long-term certified sickness. Scandinavian Journal of Public Health 35, 250-56.

Soderberg, E., Kristina, A.E. and Alexanderson, A. 2003: Sickness certification practices of physicians: a review of the literature. Scandinavian Journal of Public Health 31, 460-74.

Wahlstrom, R. and Alexanderson, K. 2004: Physicians' sicklisting practices. Scandinavian Journal of Public Health 32, (Suppl 63), 222-55. 


\section{Appendix 1: Topic Guides for GPs and Practice Managers}

\section{Introduction}

1. Background information

1.1 Catchment area

- Nature of practice population

- Unemployment levels

- Ethnic composition

1.2 GP

- Number of years as GP

- Number of years in this practice

- Hours worked in practice

- Any special interests/other roles (eg primary care trust)

- Any special interest in occupational health

- Check whether GP knows which form and can distinguish between other requests from DWP

- Estimate of number of IB113 and IB113A per month

\section{Initial reaction to $I B 113$}

Way responded when received IB113?

(explore broadly)

- How long it takes to complete the forms on average

- Which questions are difficult to answer

- Any specific problems with the IB113 (and IB113A) forms

- Views on the addition of a shorter IB113A for re-referrals and knowledge of when it should be used

- Different reaction in different circumstances? (explore) (use examples where appropriate)

- Where the form was filled in well (explore reasons)

- Where not filled in well (explore reasons)

- What could help motivation to complete IB113 well

- Extent to which it was felt that information on IB113 can influence patients' claims

Probe re: persuade/advocate/exaggerate/subjective or objective

\section{Influences}

\section{(explore)}

- Influences on the way form filled in and if filled in

Practical factors

Practice factors

Prompts:

- paperwork and whether there are any prompts to get the IB113s done

- Patient factors

Prompts:

- GP-patient relationship

- Patient's condition

- Effect on clinical management

\section{Attitudes}

(start open, then explore)

- Attitudes to importance/role of the forms

- Awareness of contractual obligation to provide this information

- Awareness of link between generation of IB113s and the sick notes they have been issuing 
- Value for whom

- GP

- practice

- patient

- DWP

- Attitudes to broader system

- Attitudes to IB/benefit assessments

- Attitudes to vocational rehabilitation

- Attitudes to role of work for health

- Attitudes to GP role in provision of medical evidence for incapacity benefit

\section{Understanding and awareness}

- Understanding of IB medical assessment process

- Understanding of purpose of IB113 and IB113A

- Understanding of what happens to information provided

- Understanding of potential impact on patients/GPs/Jobcentre Plus

- Awareness of ways DWP communicate with GPs [prompt each]

- Website

- IB204

- Desk aids

- DVD

- Medical centres (contact centres)

- Explore usefulness/reasons not used

- How did they learn about IB113 and IB113A

- Whether learning included transfer of attitudes

- Areas of understanding feel are lacking

- How would they like to receive this information

\section{Pilot communication inputs}

6.1 Mailings

(Explore whether received mailing. If no knowledge/memory - show letter)

- Receipt of

- How used

Prompts:

- Read (explore)

- Stored/filed

- Discussed with colleagues

- Views on mailing

- Content

- Format

6.2. Attendance at presentations (for respondents who attended)

- How came to attend (probe - influenced by mailing?)

- Rationale for attending

- Aspects found most/least valuable

- Information which was new

- Views on presentation

- Content

- Format

6.3. Ways communication input has changed

- Knowledge

- Understanding of IB113 systems

- Understanding of how IB113 is acted upon

Primary Health Care Research \& Development 2009; 10: 254-269 
- Understanding of potential impact on patients

- Understanding of rationale for questions on IB113

- Awareness of sources of guidance

\section{- Attitudes}

- Attitudes to role

- Whether still feel duplicating information

\section{- Behaviour}

- Approach to IB113 (probe reasons) (prompts: time taken, level of detail)

- Relations with/attitude to DWP, Jobcentre Plus, Medical Services

- Whether or not they used the website/participants pack/IB204/helpline/booked a presentation as a result of the communications strategy

- Example of changed behaviour

Probe:

- What changed

- Aspect of communications input which lead to change

- Other influences

If no/little change - explore reasons

Suggestions to improve communication inputs (explore)

\section{Suggestions}

- Any further guidance needed about IB113 and IB113A

- Preferred method of communication for guidance

- Other suggestions for improvements to informing practices about IB113s

- Suggestions about IB113 and IB113A system/procedures

- More general suggestions about how GPs can best play a role in the provision of medical evidence for incapacity benefit

Thank you

Remind about confidentiality and about honorarium

Ask to whom cheque should be made

Practice Manager Topic Guide

\section{Introduction}

\section{Background information}

\subsection{Practice}

- List size

- Number of partners

- Size of administrative team

\subsection{Practice manager}

- Number of years as PM

- Number of years in this practice

- Full or part time

- Description of role

- Other members of team with specific roles in incapacity benefit forms

- Check whether PM knows which form and can distinguish from other requests originating from DWP.

\subsection{IB113}

- Estimate number of IB113 and IB113A per month

\section{Practice process}

(Talk me through the process used in the practice for IB113)

- Who involved - roles

- Own role 
- How/when IB113 reaches GP

- Arrival - individually or batches

- Any follow up if not completed

- Any parts filled in by other people in practice

- Any role in accessing information sources (eg records, other)

- Any role in quality assurance of completion of IB113 (eg gaps, illegible)

- Any guidance used

- Circumstances when different practice system is used (explore)

- Circumstances when practice system works well

- Circumstances when practice system breaks down

- Advantages/disadvantages of practice system

IBII3a

- Ways system is different for IB113A

- Views on addition of IB113A for re-referrals

- Ways process/approach to completion differs from IB113 (probe reasons)

\section{Influences on process/system used for IB113}

(start open, then explore)

- Practical factors

- Practice factors

\section{Objectives and attitudes}

What (PMs) want to achieve by getting IB113 filled in (explore broadly)

- Different objectives in different circumstances? (explore)

General attitudes to practice role in provision of medical evidence

(for incapacity benefit)

- How that effects the way they play their part

- Has the message 'work is good for you' got across to PMs

\section{Understanding and awareness}

- Understanding of purpose of IB113 and IB113A

- Understanding of what happens to information provided

- Understanding of potential impact on patients/GPs/practice/Jobcentre Plus

- Awareness that NHS terms of contract obliges GP to complete IB113 promptly on request

- Awareness that IB113s are Dr to Dr communication (if they appear to be completing themselves)

- Areas of understanding feel are lacking

- How they would like to receive this information

- Others in practice who need to know

- How did they learn about IB113 and IB113A

- Whether learning included transfer of attitudes

- Attitude towards influence of IB113 on claims

\section{Pilot communication inputs}

\subsection{Mailings}

- Receipt of

- How used

Prompts:

- Read (explore)

- Stored/filed

- Discussed with colleagues 
- Views on mailing

- Content

- Format

6.2 Presentations (for respondents in presentation area)

Aware of practice receiving invitation to presentation - what they did with it?

Views on inputs

Prompts:

- Content

- Format/practicalities

6.3 General views on pilot communication inputs (both)

- How influenced

(Explore ways they feel communication input has changed their attitudes, knowledge or behaviour - of selves and/or practice)

Prompts:

- Approach to IB113 (probe reasons)

- Attitudes to role

- Understanding of IB113

- Awareness of sources of guidance

- Relations with/attitude to DWP, Jobcentre Plus, Medical Services

(Probe all)

- Example of changed behaviour?

Probe:

- What changed

- Aspect of communications input which lead to change

- Other influences

Suggestions to improve communication inputs (explore)

\section{Suggestions}

- Any further guidance needed about IB113 and IB113A

- Preferred method of communication

- Other suggestions for improvements to informing practices about

IB113s

- Suggestions about IB113 and IB113A forms

- Suggestions about IB113 and IB113A system/procedures

- More general suggestions about how practices can best play a role in the provision of medical evidence for incapacity benefit

Thank and remind about confidentiality 